\title{
Modeling Swarthmore spheromak reconnection experiment using hybrid code
}

\author{
Y Lin ${ }^{1,4}$, X Y Wang ${ }^{1}$, M R Brown ${ }^{2}$, M J Schaffer ${ }^{3}$ and C D Cothran ${ }^{2}$ \\ ${ }^{1}$ Physics Department, Auburn University, Auburn, AL, USA \\ 2 Department of Physics and Astronomy, Swarthmore College, Swarthmore, PA, USA \\ ${ }^{3}$ Energy Group, General Atomics, San Diego, CA, USA \\ E-mail: ylin@physics.auburn.edu
}

Received 1 November 2007, in final form 20 January 2008

Published 4 June 2008

Online at stacks.iop.org/PPCF/50/074012

\begin{abstract}
A three-dimensional (3D) hybrid-particle model is developed for investigation of magnetic reconnection in the Swarthmore Spheromak Experiment (SSX). In this numerical model, ions are treated as fully kinetic particles, and electrons are treated as a massless fluid. The plasma responds to the electromagnetic fields in a self-consistent manner. The simulation is performed in a cylindrical domain. Initially, a pair of counter-helicity spheromaks are assumed, in which the magnetic field and plasma pressure are set up according to the MHD equilibrium. The ion particles are loaded with a Maxwellian distribution function. As the simulation proceeds, magnetic reconnection takes place at the current sheet between the pair of spheromak fields. Plasma is ejected away from the $\mathrm{X}$ line towards the central axis, where heating of the transmitted ion is found. Meanwhile, quadrupole out-of-plane magnetic field structure associated with the Hall effects is present around the reconnection site. The preliminary simulation shows consistency with the SSX experiment in several aspects.
\end{abstract}

(Some figures in this article are in colour only in the electronic version)

\section{Introduction}

Magnetic reconnection is a ubiquitous process involving local annihilation of magnetic flux and global rearrangement of magnetic field lines. Reconnection occurs in solar, space and astrophysical environments but the process can also be studied in a laboratory. Reconnection results in a loss of magnetic energy with a subsequent increase in energy of ions and electrons in the plasma (flows and heating). In this paper, we compare the results of a numerical simulation with the experimental results from the Swarthmore Spheromak Experiment (SSX) [1], which include those originally presented at the IPELS 2005 and 2007 conferences.

4 Author to whom any correspondence should be addressed. 
Magnetic reconnection has been observed in the earth's magnetosphere by several satellites. In a typical scenario, parcels of magnetized plasma with oppositely directed field lines are convected into a reconnection volume (either at the magnetopause or magnetotail) at an inflow speed $V_{\text {in }}$ and ejected at a higher outflow speed $V_{\text {out }}$ which is often nearly Alfvénic. As the oppositely directed field lines convect into the reconnection volume, a current sheet is formed. If the plasma is relatively collisionless (as is generally the case in the magnetosphere) then the electron and ion fluids decouple and the current sheet thins to about the ion collisionless skin depth $\left(c / \omega_{\mathrm{pi}}\right)$. Different dynamics for electron and ion fluids generate in-plane currents and out-of-plane fields in a characteristic quadrupole structure. Evidence of two fluid kinetic effects have been observed both in the magnetopause [2] and the magnetotail [3].

Large-scale SSX dynamics have previously been modeled with a 2D axisymmetric resistive MHD code [4]. The high Lundquist number MHD code predicted much thinner current sheets than were measured in the SSX, indicating the need for a more sophisticated model. Simulated MHD field data were subsequently used to integrate test particle orbits in the SSX [5]. The test particle simulation showed a strong short duration direct acceleration of ions in the reconnection electric field followed by a weaker long-lived stochastic component associated with MHD turbulence. This result was consistent with measurements of energetic ions [6]. Recently, a 3D MHD simulation has been employed to study MHD stability of SSX plasmas [7] and non-linear stability properties of merged spheromaks [8].

In this paper, we report the development of a large-scale three-dimensional (3D) kinetic model for simulation of the SSX plasmas using a hybrid simulation code, which has been used for decades in space plasma research, including the numerical modeling of collisionless magnetic reconnection [9-12], Alfvén and various electromagnetic waves [13-15], collisionless shocks $[16,17]$ and various boundary regimes where ion kinetic physics is dominant. In particular, the code includes fully kinetic ion physics, and thus can be used to investigate the reconnection physics ranging from the scales of ion Larmor radius to global Alfvén waves. Since the fully kinetic ion physics is included self-consistently in the electromagnetics, the ion acceleration and ion heating in SSX reconnection can be studied with this model. In recent years, hybrid models have also been developed for global geometry of the magnetosphere in curvilinear [18,17] as well as the Cartesian [19] coordinate systems.

Some other efforts have also been made to simulate laboratory plasmas using an electrostatic [20] or electromagnetic [21] model with fully kinetic ion dynamics. Nevertheless, so far, the hybrid models discussed above have not been utilized in calculations for laboratory reconnection experiments. While laboratory plasmas are often confined in strong magnetic fields with a complicated geometry of flux surfaces, the SSX device, however, possesses a relatively simple geometry. The large ion beta, $\sim 1$, in the SSX indicates a ratio of ion Larmor radius to ion skin depth $\rho_{\mathrm{i}} / L_{\mathrm{i}} \sim 1$ and thus a machine's size of approximately less than a hundred $\rho_{\mathrm{i}}$. Its ions are warm with mean free path about the machine size, while electrons are cooler with shorter mean free paths. These features make it desirable that a hybrid model can be as valid for the SSX research as for space simulations. In addition to solving the fully kinetic ion particle dynamics, hybrid code is an electromagnetic code, which is suitable for the SSX reconnection with significant magnetic fluctuations $\mathrm{d} B / B$ and self-consistent wave-particle interaction.

In section 2, a description of the SSX device is provided along with a summary of recent experimental results. The 3D hybrid simulation model and the initial and boundary conditions are introduced in section 3, while the initial spheromak field is set up according to the MHD equilibrium. Preliminary results of the hybrid simulation are presented in section 4 including a direct comparison of experimental and computational diagnostics. Finally, a summary is presented in section 5 . 


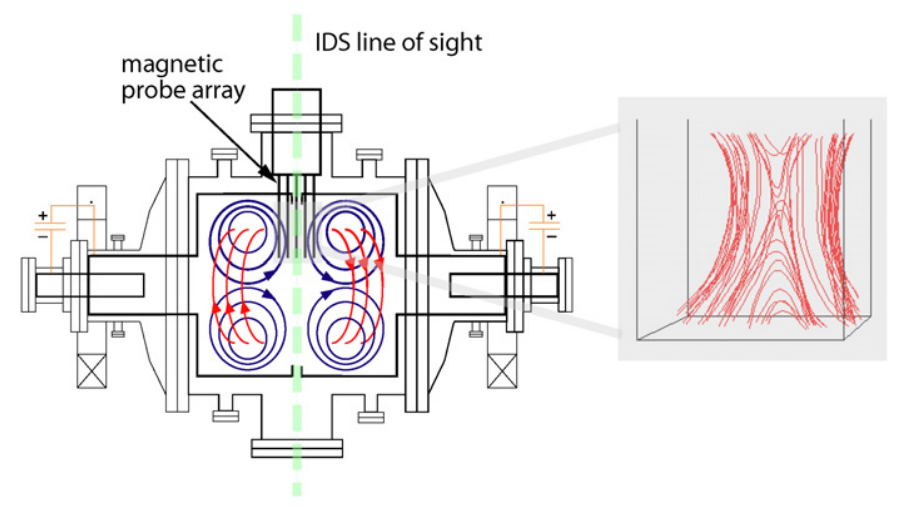

Figure 1. Swarthmore Spheromak Experiment. Schematic showing the orientation of two merging spheromaks with location of magnetic probe array and line of sight for ion Doppler spectroscopy (left). 3D magnetic data measured for merging event (right).

\section{Description of SSX reconnection experiment}

The SSX (see figure 1) is a flexible facility dedicated to the study of magnetic reconnection through the merger of nearly force-free loops of magnetized hydrogen plasma called spheromaks [1,22]. Reconnection dynamics have been studied at the SSX in a number of

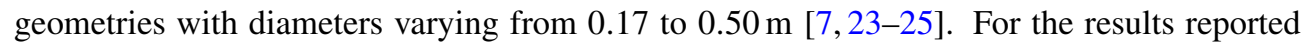
here, we study spheromaks merging in a prolate $0.4 \mathrm{~m}$ diameter, $0.6 \mathrm{~m}$ length and $3 \mathrm{~mm}$ thick wall high conductivity copper flux conserver at SSX. Merging of a pair of counter-helicity spheromaks generates turbulent 3D magnetic reconnection dynamics at the midplane.

Local and global 3D magnetic structures of SSX spheromaks have been studied with up to 600 individual internal magnetic probes operated simultaneously at $1.25 \mathrm{MHz}$ using a multiplexer system [26]. Line averaged electron density is monitored with a quadrature $\mathrm{HeNe}$ laser interferometer [27]. We can scan the electron density in a range $n_{\mathrm{e}}=(1-10) \times 10^{14} \mathrm{~cm}^{-3}$. Line averaged electron temperature is inferred from a model fit to data from a soft $\mathrm{x}$-ray array [28]. In addition, line averaged ion flow and temperature $T_{\mathrm{i}}$ at the midplane is monitored with a $1.33 \mathrm{~m}$ ion Doppler spectrometer (IDS). We achieve high spectral resolution $(0.0075 \mathrm{~nm}$ per pixel) with an Echelle grating operating at 25th order [29]. The SSX plasmas have temperatures $T_{\mathrm{i}} \cong 40-80 \mathrm{eV}, T_{\mathrm{e}} \cong 20-30 \mathrm{eV}$ for electrons, and typical magnetic fields of $0.1 \mathrm{~T}$. The plasma is fully ionized and fully magnetized with $\rho_{\mathrm{i}} \ll R$, where $R=0.2 \mathrm{~m}$ is the outer flux conserving boundary of the plasma (defined by a cylindrical copper wall). The Lundquist number $S$, the ratio of the resistive magnetic diffusion time $\tau_{\mathrm{R}}$ to the Alfvén transit time $\tau_{\mathrm{A}}$, is large for SSX, $S \cong 1000$. Accordingly, the global structure of the SSX spheromaks is fully in the magnetohydrodynamic (MHD) regime $\left(S \gg 1, \rho_{\mathrm{i}} \ll R\right)$. At $T_{\mathrm{i}}=40 \mathrm{eV}$ and $n_{\mathrm{e}}=5 \times 10^{14} \mathrm{~cm}^{-3}$, the product $\Omega_{\mathrm{i}} \tau_{\mathrm{i}} \cong 10$, where $\Omega_{\mathrm{i}}$ is the ion gyrofrequency, so a typical ion executes 10 Larmor orbits before suffering a Coulomb collision. In this case, the ion mean free path is about $0.4 \mathrm{~m}$ (the diameter of the machine) so the ions are nearly collisionless. The mean free path of $20 \mathrm{eV}$ electrons is about $0.1 \mathrm{~m}$. The ion and electron mean free paths in the SSX suggest that a hybrid code with kinetic ions and fluid electrons is a good model.

Previously, we have measured the 3D structure of magnetic reconnection [30], the production of beams of energetic ions accelerated by reconnection electric fields [6,27], and various terms in the generalized Ohm's law within the reconnecting volume [31]. The scale of the reconnection current sheet in the $\operatorname{SSX}$ is about $c / \omega_{\mathrm{pi}} \cong 2 \mathrm{~cm}$ [32], Hall effects are 
large [31] and the characteristic magnetic quadrupole is observed [7,33] leading us to believe two fluid effects are important. Local three-dimensional structures have been studied with a high resolution magnetic probe array capable of measuring vector $\boldsymbol{B}$ on a $5 \times 5 \times 8$ grid, 200 simultaneous measurements of vector $\boldsymbol{B}$, at a spatial resolution of about the ion scale $(15 \mathrm{~mm}$ radially and $18 \mathrm{~mm}$ axially which is $\leqslant c / \omega_{\text {pi }}$ in SSX) [26]. Large-scale magnetic structures have been studied at coarser resolution ( $25 \mathrm{~mm}$ resolution radially) with up to 20 linear arrays threading the machine [24]. Alfvénic outflow has been measured both with electrostatic ion energy analyzers [32] and spectroscopically [7,29].

\section{Hybrid model and initial and boundary conditions for SSX}

We now introduce the hybrid simulation model. In a hybrid code, the ions (protons) are treated as discrete particles, and the electrons are treated as a massless fluid. Quasi charge neutrality is assumed in the model. As in the usual particle-in-cell (PIC) simulations, the ion particles are advanced at their exact positions, while the electric field, magnetic field and particle moments are calculated at discretized spatial grid points. We adopt the numerical scheme of the hybrid code by Swift [18], which has been used to global-scale simulations of various processes in the magnetosphere $[9,12,17,34]$, including magnetic reconnection. In this paper, the scheme is applied to the geometry of SSX, with appropriate initial and boundary conditions. The self-consistent particle and magnetic field update method is described below.

\subsection{Numerical scheme}

In our hybrid model, the equation for ion particle motion is given as

$$
m_{\mathrm{i}} \frac{\mathrm{d} \boldsymbol{v}_{\mathrm{i}}}{\mathrm{d} t}=e \boldsymbol{E}+e \boldsymbol{v}_{\mathrm{i}} \times \boldsymbol{B}-m_{\mathrm{i}} v\left(\boldsymbol{V}_{\mathrm{i}}-\boldsymbol{V}_{\mathrm{e}}\right),
$$

where $m_{\mathrm{i}}$ is the proton mass, $\boldsymbol{v}_{\mathrm{i}}$ is the ion particle velocity, $\boldsymbol{E}$ is the electric field, $\boldsymbol{B}$ is the magnetic field, $v$ is the anomalous collision frequency between the ions and electrons and $\boldsymbol{V}_{\mathrm{e}}$ and $\boldsymbol{V}_{\mathrm{i}}$ are the bulk flow velocities of electrons and ion particles, respectively. Since the plasmas in the SSX laboratory is near collisionless, as described in section 2, in this paper, we simply impose a current-dependent anomalous collision frequency, $v / \Omega_{\mathrm{i} 0}=a J / J_{0}$, everywhere in the simulation domain, where $J$ is the current density, $a$ is a dimensionless constant, $J_{0}=B_{0} / \mu_{0} L_{\mathrm{i} 0}$ and $\Omega_{\mathrm{i} 0}, B_{0}$ and $L_{\mathrm{i} 0}$ are the ion gyrofrequency, magnetic field and ion skin depth in the ambient regions of the current sheet, respectively. For the SSX and many other reconnection experiments, the anomaly factor is large. If Alfvén time $V_{\mathrm{A}}$ is defined on the basis of an ion skin depth of $L_{\mathrm{i}} \sim 0.01 \mathrm{~m}$ in SSX, a typical Lundquist number associated with the anomalous resistivity is $S=250$, where $S=t_{\text {res }} /\left(L_{\mathrm{i}} / V_{\mathrm{A}}\right)=t_{\mathrm{res}} \Omega_{\mathrm{i}}$, and $t_{\text {res }}=1 / v$ is the resistive time scale. This, in turn, leads to $v=0.004 \Omega_{\mathrm{i}}$. Simulation runs have been performed for a peak value of $v \sim 0.001-0.2 \Omega_{\mathrm{i}}$ in the current sheet, corresponding to $a \sim 0.001-0.2$, and the results are found to be insensitive to this resistivity. No Coulomb collision is included in the present model.

The ion number density $N_{\mathrm{i}}$ and ion flow velocity $\boldsymbol{V}_{\mathrm{i}}$ are obtained in grid cells by integrating the particle moments in discrete velocity space. The electron number density $N_{\mathrm{e}}=N_{\mathrm{i}} \equiv N$ due to the charge neutrality. Since the physics to be resolved has characteristic frequency $\omega \sim \Omega_{\mathrm{i}} \ll \Omega_{\mathrm{e}}$, where $\Omega_{\mathrm{e}}$ is the electron gyrofrequency, the electric field can be obtained from the electron momentum equation

$$
\boldsymbol{E}=-\boldsymbol{V}_{\mathrm{e}} \times \boldsymbol{B}-(1 / N e) \nabla p_{\mathrm{e}}-v\left(m_{\mathrm{i}} / e\right)\left(\boldsymbol{V}_{\mathrm{e}}-\boldsymbol{V}_{\mathrm{i}}\right) .
$$


An energy equation, or equation of state, is required for electron pressure $p_{\mathrm{e}}$. For simplicity, the electron fluid is assumed to be isothermal for now, with

$$
T_{\mathrm{e}}=\text { const, }
$$

and thus $\nabla p_{\mathrm{e}}=T_{\mathrm{e}} \nabla N$. The electron flow velocity is evaluated from Ampere's law,

$$
\boldsymbol{V}_{\mathrm{e}}=\boldsymbol{V}_{\mathrm{i}}-\frac{\nabla \times \boldsymbol{B}}{\mu_{0} N e} .
$$

The magnetic field is advanced in time from Faraday's law

$$
\frac{\partial \boldsymbol{B}}{\partial t}=-\nabla \times \boldsymbol{E}
$$

A leapfrog scheme is used for particle advance, and a predictor-corrector method is used for the magnetic field update [18]. The calculation is accurate to second order in both time and space.

\subsection{Simulation domain and grid layout}

The above equations are solved in a 3D cylindrical domain, as in the SSX device. The cylinder has length $L=60 \mathrm{~cm}$, and cross-section radius $r=R=20 \mathrm{~cm}$. The simulation scheme may be solved using the Cartesian or curvilinear coordinate system. The Cartesian coordinates are utilized in this paper. Discretized spatial grids are uniformly distributed along the $x, y$ and $z$ coordinates, where $x$ and $y$ coordinates are in the cross-sectional plane of the cylinder, and $z$ is along the central axis. As described by Swift [18], Faraday's law is solved for magnetic field using the flux conservative form, which guarantees $\nabla \cdot \boldsymbol{B}=0$ as long as this condition satisfied initially. In order to solve the field equations in their integral forms, in the general structure of the code, both covariant and contravariant components are involved for the fields and the flow velocity. Correspondingly, two sets of grid cells are used, the main cells and the dual cells, in which different field quantities are centered. Note that in the Cartesian coordinate system, the metric tensor is reduced to an identity matrix. The code also implements sub-stepping loops for magnetic field update, which saves computation time.

Since the grid cells are cubic boxes in the entire domain, the grids at the side boundary of the cylindrical domain do not follow exactly the circle at $r=R=20 \mathrm{~cm}$. The boundary surface takes a zigzag shape around $r=20 \mathrm{~cm}$.

The ion skin depth is chosen as $L_{\mathrm{i}}=c / \omega_{\mathrm{pi}}=0.9 \mathrm{~cm}$ around the initial current sheet region with anti-parallel magnetic field components. This number is also approximately the initial ion skin depth throughout the domain. The magnetic field strength is about $300 \mathrm{G}$ in the ambient regions of the current sheet, corresponding to an ion gyrofrequency $\Omega_{\mathrm{i}}=e B / m_{\mathrm{i}} \simeq 2.9 \times 10^{6} \mathrm{~s}^{-1}$ and a gyroperiod of $2.2 \mu \mathrm{s}$. The minimum ion gyroperiod in the domain is about $0.45 \mu \mathrm{s}$. The ion plasma beta $\beta_{\mathrm{i}} \simeq 2$, and the Alfvén speed $V_{\mathrm{A}} \sim v_{\text {thi }} \sim 25 \mathrm{~km} \mathrm{~s}^{-1}$ around the initial current sheet, where $v_{\text {thi }}$ is the initial ion thermal speed. The ion Larmor radius $\rho_{\mathrm{i}} \simeq 0.2-9 \mathrm{~cm}$ in the whole domain. These numbers are selected according to the typical values in the SSX experiment.

The grid sizes are selected according to the physical scale lengths. In the preliminary run, the grid sizes $\Delta x=0.4 \mathrm{~cm}, \Delta y=0.4 \mathrm{~cm}$ and $\Delta z=0.94 \mathrm{~cm}$. The corresponding grid dimensions are $n x \times n y \times n z=100 \times 100 \times 65$. The particle time step is chosen as $\Delta t=0.002 \mu \mathrm{s}$.

\subsection{Initial and boundary conditions}

The chosen initial plasma state is an idealized approximation of the experimental conditions at the start of the spheromak merging reconnection. The model consists of two axisymmetric 
spheromak compact toroids placed pole to pole in a perfectly conducting circular cylinder flux conserver with closed ends. Each spheromak is individually in finite-beta, single-fluid magneto static equilibrium. The spheromaks are identical, except that one has a right-handed and the other a left-handed magnetic field, thus setting up the pair for a counter-helicity merge. The spheromak toroidal currents are in the same direction, as in the experiment, to promote prompt reconnection.

The spheromak CT is a computed solution to the Grad-Shafranov (GS) equilibrium equation, and the other spheromak is constructed from it by appropriate reflections. The GS equation is solved numerically by the finite element code EQLFE [35], when solves the GS equation in a right circular cylinder computational domain corresponding to one half of the SSX flux conserver. The plasma gun is not modeled in detail, but the gun magnetizing flux, which is applied so slowly in the experiment that it penetrates the gun electrodes and the flux conserver, is calculated and treated as a frozen flux boundary condition for the GS solution. The boundary condition at the $z=0$ symmetry plane is $B_{z}=0$. The total toroidal plasma current in one spheromak, $42 \mathrm{kA}$, is adjusted until the computed solution approximates the measured magnetic field, which is $B_{z} \simeq 0.10 \mathrm{~T}$ at the cylindrical surface far from the ends and from $z=0$. The normalized parallel current $J_{\|} / B$ is distributed proportionally to $\psi^{3 / 2}$, where the poloidal magnetic flux $\psi$ runs from 0 at $r=0$ to its maximum value $\psi_{\text {axis }}$ at the magnetic axis. This choice sets $J=0$ at the plasma boundary without being unrealistically peaked on axis. For comparison, the fully relaxed Woltjer-Taylor current distribution is $J_{\|} / B \sim \psi$.

The plasma total (electron plus ion) pressure is distributed as $p=p_{\text {edge }}\left[1+\left(\psi / \psi_{\text {axis }}\right)^{1.75}\right]$. The pressure distribution in the experiment is not well known. For the present calculations, the peak pressure at the magnetic axis is $2.44 \mathrm{kPa}$, the local magnetic field $\boldsymbol{B}$ is purely toroidal and is $0.141 \mathrm{~T}$ and the local $\beta=p_{\text {peak }} /\left(B^{2} / 2 \mu_{0}\right)=0.31$. The large edge pressure was selected arbitrarily to avoid a low mass density edge and the resulting small numerical time steps in the subsequent dynamical simulation. This initial condition pressure may be divided among electron and ion density and temperatures.

Note that our initial conditions are an idealized simplification of the experimental time when two plasmas, injected through apertures in the opposite ends of the flux conserver, meet at the midplane. The data of the SSX do show a period after the plasma encounter when there are two slightly connected spheromaks, consistent with our initial configuration. The initial conditions of magnetic field and plasma pressure described above have also been used in an earlier MHD simulation of SSX [8].

Initially, the ions are loaded with a Maxwellian distribution. The initial currents are assumed to be carried by electrons. In the PIC scheme, the particles are macro particles accelerated by weighted fields on the ambient discrete grids. To assure a good statistics in the integration for particle moments, about 150 particles are loaded in each grid cell, corresponding to a density of $7.5 \times 10^{14} \mathrm{~cm}^{-3}$ in the real unit. A total of $\sim 5 \times 10^{8}$ particles are used in a run. Figure 2 shows magnetic field lines in the partial domain in 3D perspective and ion density contours at $z= \pm 15 \mathrm{~cm}$ and $z=0$. The initial current sheet is located at $z=0$.

Conducting boundary conditions are applied to all the boundaries, at $z= \pm L / 2= \pm 30$ and $r=R=20 \mathrm{~cm}$. At all these boundaries, the normal component of magnetic field $\boldsymbol{B}_{n}$ is fixed at the initial values, and the tangential components of electric field $\boldsymbol{E}_{t}=0$. The ion particles are perfectly reflected at the boundaries, with the outgoing normal velocity $\boldsymbol{v}_{\text {ir }}$ of particle reversed to $-\boldsymbol{v}_{\text {ir }}$, while the tangential velocity $\boldsymbol{v}_{\text {it }}$ is unchanged.

The hybrid scheme itself has been well used in space plasma simulations. Our benchmark of the hybrid scheme for a uniform plasma showed perfect consistency between the linear dispersion relations obtained from the simulation and the fully kinetic linear theory for $k \rho_{\mathrm{i}} \sim 1$ and $\omega \sim \Omega_{\mathrm{i}}$. To apply the scheme to the device of the SSX, the only non-trivial modification 


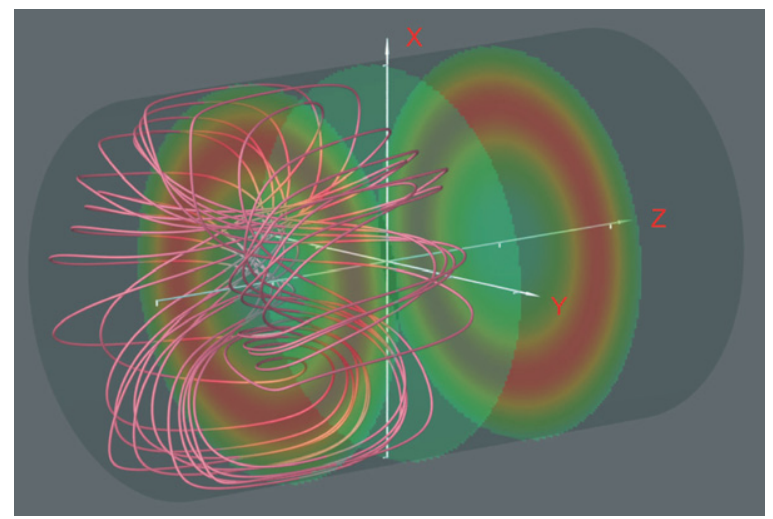

Figure 2. Initial magnetic field lines in the partial cylindrical simulation domain in $3 \mathrm{D}$ perspective and cross-sectional cuts of ion density contours.

of the code is the handling of boundary conditions at $r \sim R$. We let particles be reflected at exactly $r=R$, while the boundary grids for $\boldsymbol{E}$ and $\boldsymbol{B}$ fields reside on the actual zigzag boundary grid lines. The normal and tangential components of both particles and fields are projected relative to the cylindrical surface of $r=R$, to allow the execution of the boundary conditions.

In order for particles near $r=R$ to see fields weighted from all four ambient grids in the cross-sectional planes of constant $z$, the field quantities are extrapolated to an extra grid point outside the $r=R$ circle. On the other hand, the particle moments, e.g. the density and bulk flow velocity, at the field boundary grids are calculated by integration in the discrete phase space in partial cells within $r \leqslant R$ and then weighted to the full cell. We find that the handling of particle density at the boundary cells is still imperfect. The noise level of $N$ at the boundary is higher than inside the domain. In the preliminary run, the noise grows into instability at $t>4 \mu \mathrm{s}$. This is an area to be improved technically, so that the calculation can be done for a longer period in the future.

\section{Initial results}

In this section, we briefly show the results obtained from a preliminary run using the hybrid model described above. The simulation is conducted by parallel computation using message passing interface (MPI), with both domain decomposition and particle domain parallelization.

Figure 3 presents magnetic field lines and ion density contours in the $y=0$ plane at $t=2.1 \mu \mathrm{s}$. Reconnection is seen at the $\mathrm{X}$ line in two places, at $x \simeq+12$ and $-10 \mathrm{~cm}$ in this plane near $z=0$, in the current sheet between the left and the right spheromaks. Thus, the beginning of non-axisymmetric 3D behavior is seen even at this early time. Due to the initial symmetry in the toroidal direction, the reconnection $\mathrm{X}$ line is curved along a near circular line. The ions are transmitted along the reconnected fluxes from the two sides of the current sheet, and ejected away from the $\mathrm{X}$ line. The presence of bi-directional flows along a diameter chord has been recorded in the SSX reconnection experiments [25]. The radial size of the outflow region is $\sim 5 \mathrm{~cm}$, which corresponds to an Alfvén convection time and particle thermal transmission time of $\sim 2 \mu \mathrm{s}$. The accumulation of the transmitted ions leads to a local density concentration near the central axis at $x=0$, where $N$ is enhanced by nearly 3 times from the initial value. Field line traces around the reconnection region in $x>0$ are presented in 


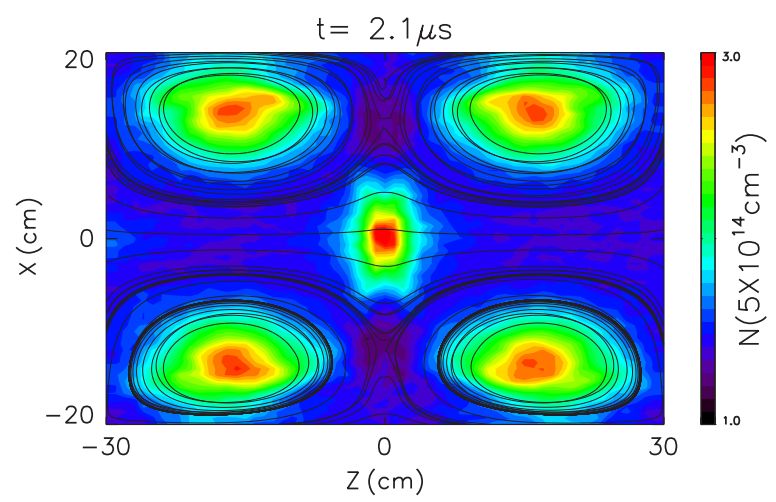

Figure 3. Field lines and ion density contours in the $y=0$ plane at $t=2.1 \mu \mathrm{s}$. The origin of the coordinate system is at the center of the cylindrical domain, while the $z$ axis is the central axis. Magnetic reconnection is seen at two locations around $(x, z) \simeq(+12 \mathrm{~cm}, 0)$ and $(-10 \mathrm{~cm}, 0)$.

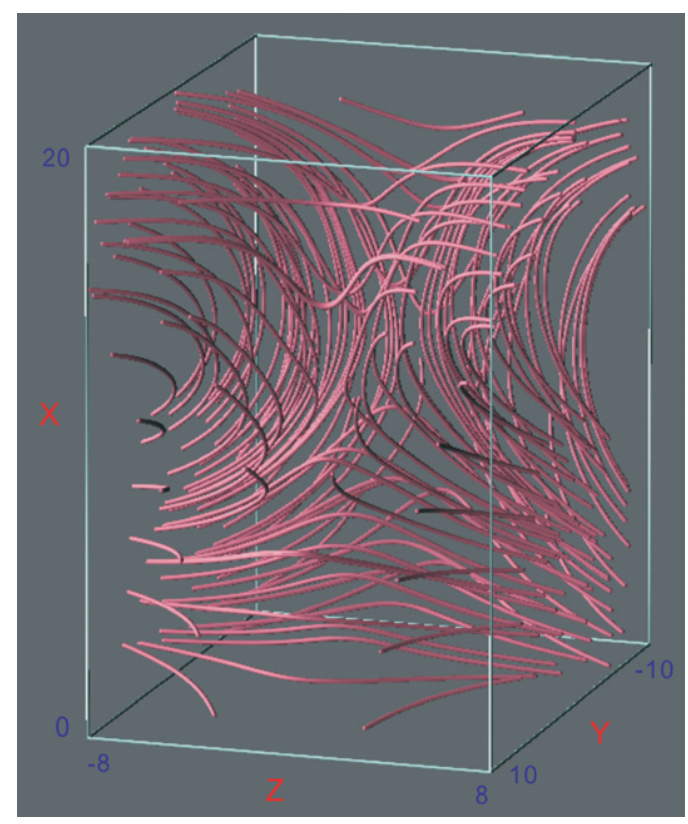

Figure 4. Field line traces around a reconnection region showing 3D magnetic field topology of reconnection.

figure 4. The simulated field lines show a complicated, 3D topology, as observed in the SSX shown in the figure 1 inset.

The ion bulk flow moment is shown in figure 5 for $t=1.46 \mu \mathrm{s}$, when the strongest flow ejection is seen during the reconnection. The left plot of figure 5 depicts contours of the radial component of flow, $V_{r}$, in the $y=0$ plane, and the right plot shows $V_{r}$ at $z=0$ in the crosssectional $(x y)$ plane. The poloidal field lines at this moment are also superposed onto the left plot to illustrate the locations of the $\mathrm{X}$ line. Inward ion flow ejections towards the $x=0$ axis are seen in both planes, in the outflow regions of the two reconnection sites. On the outward side of either locations of $\mathrm{X}$ line, however, no apparent ion flow is found. Meanwhile, toroidal flows (not shown) are also produced in the outflow region of reconnection, and get stronger at 


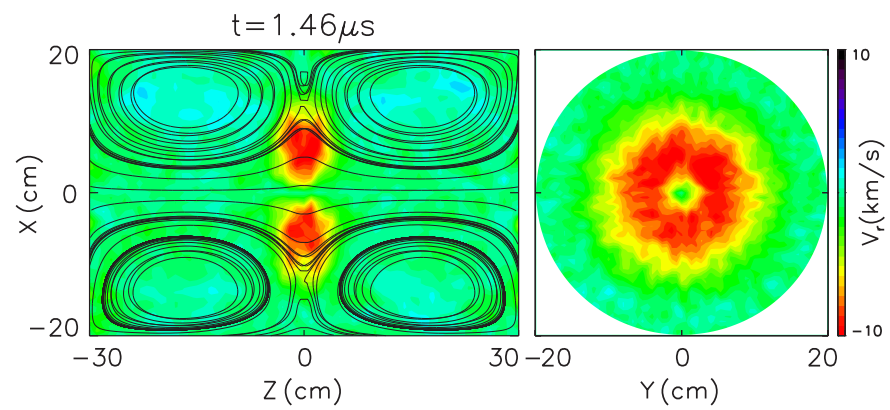

Figure 5. Radial component of ion bulk flow velocity $V_{r}$ and poloidal field lines in the $y=0$ plane (left plot) and $V_{r}$ in the $z=0$ plane (right) at $t=1.46 \mu \mathrm{s}$.
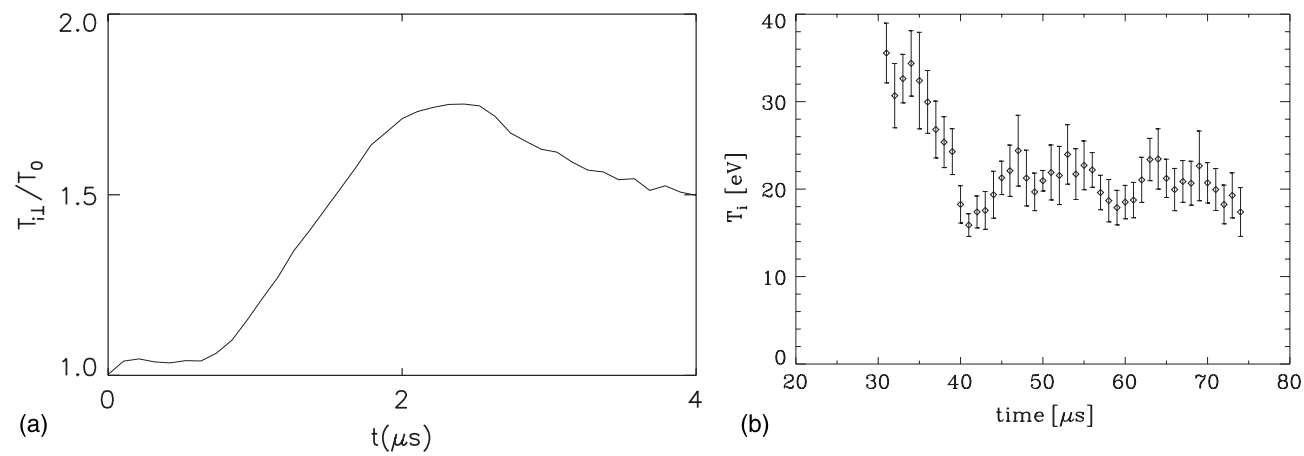

Figure 6. (a) Time variation of ion temperature ratio $T_{\mathrm{i} \perp} / T_{0}$ obtained in the simulation, where $T_{0}$ is the initial temperature. $(b)$ Ion temperature measured in the SSX reconnection experiment.

later times. In the $x y$ plane, the flow occupies a region of thick ring, with $V_{r}$ stops at $r \sim 1 \mathrm{~cm}$. There also appear to be multi-dimensional wave activities in the $V_{r}$ pattern. In later times, e.g. at $t=2 \mu \mathrm{s}, V_{r}$ in the toriodal plane is seen to break up into finer structures with higher wave number $k$.

In addition, ion heating is found in the simulation. During the short course of the run, the heated ions are located mainly where the enhanced ion density is observed, at the center of the cylindrical domain. The Larmor radius of these heated ions is about $1-2 \mathrm{~cm}$. Figure $6(a)$ shows the ion temperature ratio $T_{\mathrm{i} \perp} / T_{0}$ as a function of time $t$, where $T_{\mathrm{i} \perp}$ is the peak of the perpendicular ion temperature in the reconnection, and $T_{0}=5 \mathrm{eV}$ is the initial ion temperature. The heating appears mainly in the direction perpendicular to the magnetic field. The temperature $T_{\mathrm{i} \perp}$ is seen to increase by a factor of 1.76, and then start to drop at $t>2 \mu \mathrm{s}$. On the other hand, the ion temperature measured in the SSX reconnection experiment is plotted in figure $6(b)$, which shows the period during which the temperature drops from its maximum value during the reconnection to a lower background value in the subsequent reconnected state. The ratio of the peak to background temperatures obtained in the simulation is consistent with the lab experiment. Note that the time $t=0$ in the simulation corresponds to $t$ just before $30 \mu \mathrm{s}$ in the experiment. The experimental data is not shown for $t<30 \mu$ s while the two cool spheromaks have not arrived yet. After the reconnection is over, the temperature cools back, and the final $T_{\mathrm{i}}$ in figure $6(b)$ is that of unheated plasma of single spheromak [7]. 


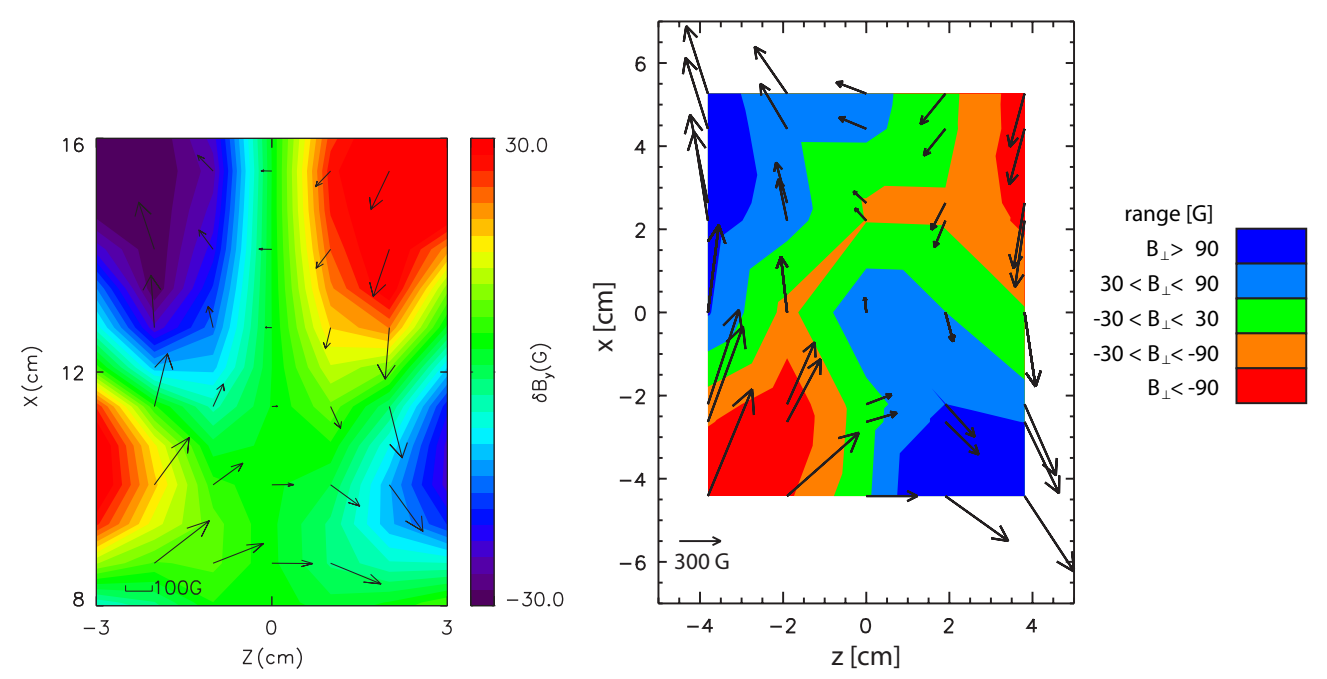

Figure 7. (a) Change of toroidal field component $B_{y}$ relative to the initial value in the $y=0$ plane at $t=3.0 \mu \mathrm{s}$. The arrows indicate the projected $\boldsymbol{B}$ field. (b) $B_{y}$ measured in a SSX reconnection event.

Finally, figure $7(a)$ depicts $\delta B_{y}$, the change of the $B_{y}$ component of the magnetic field relative to the initial value, in the $y=0$ plane at $t=3.0 \mu \mathrm{s}$. This $\delta B_{y}$ is equivalent to the perturbation of the toroidal field component in the SSX. The field vectors projected to the $y=0$ plane are shown by arrows in figure 7(a). Within the plotted region, $|z| \sim 3 L_{\mathrm{i}}$ around the $\mathrm{X}$ line, the anti-parallel magnetic components are dominated by the poloidal fields in the plane. A quadrupole structure is seen in the out-of-plane field, $\delta B_{y}$, around the $\mathrm{X}$ line. The generation of the $\delta B_{y}$ is due to the Hall effects [36,37] associated with the ion inertia. The senses of $\delta B_{y}$ in the quadrupole structure are consistent with the senses of the Hall current loops in the current layer, and the quadrupole structure is consistent with the $B_{y}$ measured in the SSX reconnection event [33], as shown in figure 7(b).

\section{Summary}

In summary, we have developed a first hybrid model for simulation of the SSX plasmas. The 3D model resolves fully kinetic ion dynamics in a self-consistent electromagnetic field. The simulation is carried out for a cylindrical domain containing a pair of counter-helicity spheromaks, as in the real SSX device. To set up the initial static condition, equilibrium structure of a pair of counter-helicity spheromaks in the SSX are solved. Merging of the initial force-free fields is obtained in the dynamic simulation. The initial results of the simulated SSX reconnection events show the presence of outflow ejections from the $\mathrm{X}$ line and the Hall magnetic field structure. In addition, perpendicular ion heating is found in the ions that are transmitted from the spheromaks through the reconnected field lines and ejected to the central axis. The presence of the bi-directional flows, Hall magnetic field structure and ion heating agrees quite well with the SSX reconnection experiments.

The code is developed by applying the hybrid scheme that has been used in space plasma simulations to the SSX geometry. In this sense, this paper addresses a collaboration among researchers of space and laboratory modeling and laboratory experiment, and reports an initial progress of the collaboration. 
The preliminary results shown in this paper only correspond to an initial stage of the merging. In order to further investigate the local and global physics of the SSX reconnection and compare the simulation with the experiment, the code needs to be improved for handling of the boundary conditions so that a longer run can be achieved. Although not long enough to see the dominant 3D effects, the simulation has indicated the presence of non-axisymmetric structure. The SSXs have also found the development of the non-axisymmetric structures as time proceeds.

In the present simulation, collision effects are simply modeled as the anomalous resistivity between electrons and ions. Since the Coulomb collision frequency is much smaller than the ion gyrofrequency in the SSX reconnection experiment, our present collision model should be a valid approximation. In the future simulations, the Coulomb collision can be added if necessary using a Monte Carlo method, and the effects of collision on reconnection can be investigated.

\section{Acknowledgments}

The research at Auburn University was supported by DoE grant DE-FG02-05ER54826. The research at Swarthmore College and General Atomics was supported by DoE grant DE-FG0200ER54604. Computer resources were provided by the NERSC supercomputer center and the Arctic Region Supercomputer Center.

\section{References}

[1] Brown M R 1999 Phys. Plasmas 61717

[2] Mozer F S, Bale S D and Phan T D 2002 Phys. Rev. Lett. 8915002

[3] Wygant J R et al 2005 J. Geophys. Res. 110 A09206

[4] Lukin V S, Qin G, Matthaeus W H and Brown M R 2001 Phys. Plasmas 81600

[5] Qin G, Lukin V S, Cothran C D, Brown M R and Matthaeus W H 2001 Phys. Plasmas 84816

[6] Brown M R et al 2002 Phys. Plasmas 92077

[7] Brown M R et al 2006 Phys. Plasmas 13102503

[8] Belova E V, Davidson R C, Ji H, Yamada M, Cothran C D, Brown M R and Schaffer M J 2006 Nucl. Fusion 46162

[9] Lin Y and Swift D W 1996 J. Geophys. Res. 10119859

[10] Karimabadi H, Krauss-Varban D, Omidi N and Vu H X 1999 J. Geophys. Res. 10412313

[11] Nakamura M and Scholer M 2000 J. Geophys. Res. 10523179

[12] Lin Y 2001 J. Geophys. Res. 10625451

[13] Swift D W and Lee L C 1983 J. Geophys. Res. 88111

[14] Winske D and Quest K B 1986 J. Geophys. Res. 918789

[15] Wang X Y and Lin Y 2003 Phys. Plasmas 103528

[16] Winske D, Omidi N, Quest K B and Thomas V A 1990 J. Geophys. Res. 9518821

[17] Lin Y and Wang X Y 2005 J. Geophys. Res. 110 A12216

[18] Swift D W 1996 J. Comput. Phys. 126109

[19] Omidi N, Blanco-Cano X and Russell C T 2005 J. Geophys. Res. 110 A12212

[20] Sherlock M, Chittenden J P, Lebedev S V and Haines M G 2004 Phys. Plasmas 111609

[21] Belova E V, Jardin S C, Ji H, Yamada M and Kulsrud R 2000 Phys. Plasmas 74996

[22] Bellan P M 2000 Spheromaks (London: Imperial College Press)

[23] Geddes C G R, Kornack T W and Brown M R 1998 Phys. Plasmas 51027

[24] Cothran C D, Falk A, Fefferman A, Landreman M, Brown M R and Schaffer M J 2003 Phys. Plasmas 101748

[25] Brown M R, Cothran C D and Fung J 2006 Phys. Plasmas 13056503

[26] Landreman M, Cothran C D, Brown M R, Kostora M and Slough J T 2003 Rev. Sci. Instrum. 742361 
[27] Brown M R, Cothran C D, Landreman, Schlossberg M D and Matthaeus W H 2002 Astrophys. J. Lett. 577 L63

[28] Chaplin V, Horwitz J, Brown M R, Cohen D H and Cothran C D 2008 Phys. Plasmas submitted

[29] Cothran C D, Fung J, Brown M R and Schaffer M J 2006 Rev. Sci. Instrum. 77063504

[30] Cothran C D, Landreman M, Matthaeus W H and Brown M R 2003 Geophys. Res. Lett. 301213

[31] Cothran C D, Landreman M, Brown M R and Matthaeus W H 2005 Geophys. Res. Lett. 32 L03105

[32] Kornack T W, Sollins P K and Brown M R 1998 Phys. Rev. E 58 R36

[33] Matthaeus W H, Cothran C D, Landreman M and Brown M R 2005 Geophys. Res. Lett. 32 L23104

[34] Lin Y and Wang X Y 2006 Geophys. Res. Lett. 33 L21104 doi:10.1029/2006GL027736

[35] Leuer J A, Schaffer M J, Parks P B and Brown M R 2002 Bull. Am. Phys. Soc. 47275

[36] Sonnerup B U O 1979 Solar System Plasma Physics ed L T Lanzerotti et al (Amsterdam: North Holland) vol 3 p 45

[37] Shay M A, Drake J F, Rogers B N and Denton R E 2001 J. Geophys. Res. 1063759 